\title{
Neurocognitive Performance: Returning to Competition
}

\author{
Larry W. McDaniel \\ Department of Physical Education \& Exercise Science \\ Dakota State University \\ E-mail: larry.mcdaniel@dsu.edu \\ Kyle McIntire \\ Student of Exercise Science \\ Dakota State University \\ E-mail: kyle.mcintire@dsu.edu
}

\begin{abstract}
Athletes who suffer from concussions under report their symptoms in order to expedite their return to competition. Athletic trainers and coaches must be aware of what is going on with athletes, even if it means requiring them to refrain from competition. Ninety percent of concussions are minor and can be difficult to diagnosis. There is a lack of guidelines available for physicians and athletic trainers to follow when dealing with concussions. However, healthcare officials are recognizing the importance of concussion management and experts agree that athletes who have concussion symptoms should not return to competition until they are fully resolved. Computerized testing can efficiently and effectively assess and diagnose a concussion. Physicians and athletic trainers can monitor these tests, which saves athletes time and money. Computerized tests such as ImPACT and CRI provide clear results that are easy to read. By reviewing the results physicians and athletic trainers may better diagnose the symptoms which prevent the athletes from being dishonest about their symptoms in order to return to competition before they have recovered.
\end{abstract}

Keywords: Concussion, Return to competition, Head injuries, Neurocognitive performance, ImPACT

\section{Purpose}

The purpose of this paper was to study concussions and determine when an athlete suffering from this injury is ready to return to competition. An additional focus will be to search for information related to athlete's neurocognitive performance after symptoms of a concussion have dissipated.

\section{Introduction for Need for Study}

Injuries happen in most sports, and when they do who is in charge of determining when the athlete can return to participation? For the most part, the athlete has too much control of when they think they can perform well enough to participate in activities. "The complexity of concussion injuries requires clinicians to use a variety of tools for information, but the current tendency is to base the return-to-play decision on the athlete's self-reporting of symptoms and ability to perform sport-specific tasks without a recurrence of concussion symptoms" (Notebaert \& Guskiewicz, 2005). Athletic trainers or coaches are responsible for making the call as to when an athlete will be permitted to return to competition. When dealing with concussions athletes must be vigilant because the risk of permanent damage is much higher than other injuries. Over the last decade there has not been a set criterion for when athletes who have suffered from a concussion may return to activity. So when is an athlete who has suffered a concussion, ready to return to participation? "Sport related concussions are a distinct clinical subset of traumatic brain injury because they are often witnessed and not usually associated with other injury. More than $90 \%$ of these injuries are of mild severity characterized by transient confusion, brief amnesia, and no loss of consciousness. Thus it is possible for an athlete to be concussed and still continue to play. Also, management decisions are easily influenced by an athlete's desire to return to competition and a coach's will to win.18 Although some traumatic brain injuries are more severe than others, none are trivial, and each has the potential to be lethal" (Benson, Rose, Meeuwisse, Kissick, \& Roberts, 2002). There is a need for continued study of these injuries because of the possibility of permanent damage concussions may cause. "Team physicians and athletic trainers increasingly are recognizing the importance of concussion management in athletes. "Although consensus has not been reached regarding the specifics of return-to-play guidelines after a concussion, experts uniformly agree that athletes should not return to play until all symptoms have resolved" (Erlanger, Saliba, Barth, Almquist, \& et al., 2001). "There are 300,000 sports related concussions per year. If an athlete returns to competition too quickly after a concussion, there is a high chance of brain damage or death if another concussion is received. Second 
Impact Syndrome, which is when a person who is recovering from a concussion gets another one, is responsible for approximately 30-40 deaths in the last decade" (ImPACT, 2009). According to the Center for Disease Control, A concussion, which is also known as a Traumatic Brain Injury (TBI), is "caused by a blow or jolt to the head or a penetrating head injury that disrupts the normal function of the brain. Not all blows or jolts to the head result in a Traumatic Brain Injuries (TBI). The severity of a TBI may range from "mild," i.e., a brief change in mental status or consciousness to "severe," i.e., an extended period of unconsciousness or amnesia after the injury" (Mitchko, Huitric, Sarmiento, Hayes, \& et al, 2007). According to Dr. Michael Collins, assistant director of the University of Pittsburgh Medical Center's (UPMC) Sports Medicine Concussion Program "The blow shakes the brain in the skull like a yolk sloshing around inside an eggshell" (McCollum, 2009). Concussions have many symptoms some of the more common symptoms that are reported by athletes are: headaches, nausea, balance problems and/or dizziness, fuzzy or double vision, sensitivity to light and/or noise, feeling sluggish or foggy, change in sleep patterns, concentration or memory problems, and unconsciousness.

\section{Methodology}

Guidelines have not been set for the assessment of concussions. "Past surveys suggest that most athletic trainers do not adhere to any specific concussion classification system or return-to-play guidelines in the clinical decision making process" (Ferrara, McCrea, Peterson, \& Guskiewicz, 2001). Doctors, coaches, and athletic trainers can use whatever type of assessment that they feel comfortable with. There are many types of tests that can be done to assess concussions such as surveys, which are basically questions that physicians or athletic trainers ask the athlete to determine how severe a concussion is, memory tests and balance tests. There are also tests that can administrated with scans which include CT, MRI, and EEG scans that will determine if an athlete has a concussion. All of these tests lead to the same conclusion, does the athlete have a concussion and how severe it is. Many schools and athletic trainers are testing the neurocognitive performance of the injured athlete. What does this mean? This means that the athlete is tested on mental process such as perception, memory, judgment, and reasoning.

In some cases, when obvious signs are not apparent, concussion symptoms may go unnoticed. This is why many medical professionals, athletic trainers, and coaches are turning to neurocognitive performance testing. Neurocognitive performance tests can not only show if the athlete is suffering from a concussion but also the recovery progress of the athlete. Another important key factor is that these tests diagnosis if the athlete is not telling the truth about their recovery process in order to return to competition. "Recently, the practicality of eliminating or delaying neurocognitive testing in cases of "simple" concussion has been questioned. One group recommended self-reported symptoms be used to the exclusion of other measures, in large part because neurocognitive decrements have not been shown to persist beyond symptom resolution. In contrast, others suggested that neurocognitive testing should be implemented soon after the concussion diagnosis due to the potential for an athlete to underreport concussion-related symptoms in order to expedite return to play" (Broglio, Macciocchi, \& Ferrara, 2007). These tests can bring into perspective is if an athlete is truly healthy enough to return to competition after the symptoms are reduced. "We are uncertain whether concussed athletes who no longer report post concussion symptoms continue to show neurocognitive impairment related to their injuries" (Broglio, \& et al., 2007).

There are different types of computerized tests that are available for athletic trainers and other health professionals to use. These tests are taken on computers over the internet. After a test is taken a chart is produced which is reviewed by a professional. To use these computerized tests athletes must first take a baseline test to evaluate where their neurocognitive performance is when healthy. If a concussion is received the athlete retakes the test to find out the severity. Before the athlete is able to return to competition, they must retake the tests until their neurocognitive performance is at a level that is within the limits of their baseline score. After the athlete receives a concussion the number will spike up indicating the severity of the injury. After the concussion, the athlete must refrain from activity until his/her overall score is back to the baseline score. An advantage that these computerized tests offer is that the diagnosis takes a short period of time. "CRI (Concussion Resolution Index) baseline assessments take less than 25 minutes, and post concussion assessments take approximately 20 minutes" (Erlanger, \& et al., 2001). Going to a professional to administer this test would take much longer. "Implementing traditional neuropsychological protocols is time consuming, inefficient, and expensive. Athletes must be tested individually in a face-to-face format; testing requires 30 minutes to 2 hours per athlete; administration, scoring, and statistical analysis must be carried out by trained clinicians; and, in the event of a concussion, follow-up tests must be scheduled, administered, scored, and statistically analyzed. All of these factors allow the athletic trainer's the ability to use the results in a timely fashion" (Erlanger, \& et al., 2001). 
In a recent study, the ImPACT test was used to examine the effects of sports-related concussions. Athletes were administered a baseline test before competition started. "Our concussion battery includes the Symptom Assessment Scale (SAS) and the ImPACT (ImPACT Applications, Inc, Pittsburgh, PA) neurocognitive assessment for concussion" (Broglio, \& et al, 2007). As practice and competition got under way, anyone who suffered a concussion clinically diagnosed by a physician completed a series of tests. "During the self-reported symptomatic (SRS) period after injury, the athlete's symptoms were monitored and recorded daily on the SAS. On the day the athlete self-reported being asymptomatic (SRA), the complete assessment battery was re-administered" (Broglio, \& et al, 2007). For the athlete's data to be used in the analysis they had to meet three criteria. They first had to complete the baseline test which was administered immediately then the athletes were diagnosed to determine if a concussion was found and within 72 hours follow up exam is administered. The third requirement athletes had to meet was to determine if the severity of the symptoms at the SRA assessment point. "After complete injury resolution, each athlete was classified as having sustained a simple or complex concussion based on the guidelines provided in the summary and agreement statement of the 2nd International Conference on Concussion in Sport. A simple concussion was characterized by no loss of consciousness at the time of injury and symptom resolution within 10 days of injury. A complex concussion was characterized by a loss of consciousness lasting longer than 1 minute at the time of injury or symptoms persisting beyond 10 days after injury" (Broglio, \& et al, 2007).

\section{Findings}

"ImPACT takes approximately 20 minutes to complete. This computer program measures multiple aspects of cognitive functioning in athletes including: attention span, working memory, sustained and selective attention time, responsive variability, non-verbal problem solving, and reaction time" (ImPACT, 2009). This test is comprised of six modules that when mathematically combined produces composite scores for verbal memory, visual memory, visual-motor speed, and reaction time. "The computer test also includes an evaluation of concussion-related symptoms. On a 22 -item symptom list, the athlete ranks each item for current severity on a Likert scale (0 to 6); the maximum total score is 132" (Broglio, \& et al, 2007). According to the study, 21 NCAA Division I athletes met the criteria. There were 16 males and 5 females, whose average age was 19.81 years, +/1.25 years; the average previous concussions were $1.76,+/-2.02$. Out of the 21 athletes, $17(81 \%)$ were shown to have simple concussions, and $4(19 \%)$ were shown to have complex concussions. "Of all the concussed athletes included in the analysis, $15(71 \%)$ were identified as impaired according to the RCI calculations on at least 1 ImPACT cognitive variable at the SRS assessment relative to baseline. An additional 2 athletes were identified as impaired on the ImPACT symptom inventory, for a total of 17 athletes (81\%) identified as impaired on at least 1 cognitive or symptom variable. Impairment on specific variables included 7 athletes on verbal memory, 8 on visual memory, 5 on visual-motor speed, and 9 on reaction time" (Broglio, \& et al, 2007). In this study there were eight athletes who no longer reported any concussion related symptoms. When data from the ImPACT test were reviewed it showed that these athletes continued to have impairments on at least one variable when compared to their baseline test.

\section{Conclusion}

Concussions may be difficult to identify since more than $90 \%$ of concussions are of mild severity which make them difficult to diagnose. Coaches and athletic trainers may become caught up in the emotions of the game and overlook the athlete's symptoms. These professionals need to be alert for signs and symptoms that athletes may be experiencing which range from looks of confusion, forgetfulness, unconsciousness, and amnesia. Concussions are injuries to the brain and if they do not receive medical attention they can cause serious brain damage or death. Post concussion syndrome may lead to ailments such as memory loss and frequent headaches which may develop into various other disabilities.

New ways of assessing and diagnosing concussions are available. Computerized programs are becoming more reliable and time effective. Visiting a physician can be expensive and time consuming, however, with the new computerized programs such as the ImPACT and CRI tests assessing and diagnosing a concussion takes a matter of minutes. With these modules, physicians and athletic trainers may efficiently and effectively handle a concussion and know when the athlete can safely return to competition.

\section{References}

Benson B. W., Rose M. S., Meeuwisse W. H., Kissick J. \& Roberts W. O. (2002). The impact of face shield use on concussions in ice hockey: A multivariate analysis / Commentary. British Journal of Sports Medicine, 36(1), 27-32. Retrieved September 11. 2009, from Research Library. (Document ID: 109473366). 
Broglio, S., Macciocchi, S. \& Ferrara, M. (2007). Neurocognitive Performance of Concussed Athletes When Symptom Free. Journal of Athletic Training, 42(4), 504-508. Retrieved September 11, 2009, from Research Library. (Document ID: 1410176691).

Erlanger D., Saliba E., Barth J., Almquist J. \& et al. (2001). Monitoring resolution of post concussion symptoms in athletes: Preliminary results of a web-based neuropsychological test protocol. Journal of Athletic Training, 36(3), 280-287. Retrieved September 11, 2009, from Research Library. (Document ID: 84843118).

Ferrara S., McCrea M., Peterson C. L. \& Guskiewicz K. M. (2001). A survey of practice patterns in concussion assessment and management. Journal of Athletic Training, 36(2), 145-149. Retrieved September 11, 2009, from Research Library. (Document ID: 77223309).

ImPACT. (2006-2009). Home Page. Retrieved October 21, 2009, from ImPACT test Web site: http://www.impacttest.com/

McCollum, S. (2009, April). The Invisible Injury. Scholastic Choices, 24(6), 16-19, T7. Retrieved September 11, 2009, from Research Library. (Document ID: 1665741941).

Mitchko J., Huitric M., Sarmiento K., Hayes G. (2007). et al American Journal of Health Education; Mar/Apr 38, 2; ProQuest Education Journals pg. 99

Notebaert, A. J. \& Guskiewicz, K. M. (2005). Current Trends in Athletic Training Practice for Concussion Assessment and Management. Journal of Athletic Training, 40(4), 320-325. Retrieved September 11, 2009, from Research Library. (Document ID: 957212201). 